\title{
Application of transmission-line super theory to classical transmission lines with risers
}

\author{
R. Rambousky ${ }^{1}$, J. Nitsch ${ }^{2}$, and S. Tkachenko ${ }^{2}$ \\ ${ }^{1}$ Bundeswehr Research Institute for Protective Technologies and NBC Protection (WIS), Munster, Germany \\ ${ }^{2}$ Otto-von-Guericke University Magdeburg, Magdeburg, Germany \\ Correspondence to: R. Rambousky (ronald.rambousky@ieee.org)
}

Received: 5 November 2014 - Revised: 22 April 2015 - Accepted: 4 May 2015 - Published: 3 November 2015

\begin{abstract}
By applying the Transmission-Line Super Theory (TLST) to a practical transmission-line configuration (two risers and a horizontal part of the line parallel to the ground plane) it is elaborated under which physical and geometrical conditions the horizontal part of the transmission-line can be represented by a classical telegrapher equation with a sufficiently accurate description of the physical properties of the line. The risers together with the part of the horizontal line close to them are treated as separate lines using the TLST. Novel frequency and local dependent reflection coefficients are introduced to take into account the action of the bends and their radiation. They can be derived from the matrizant elements of the TLST solution. It is shown that the solution of the resulting network and the TLST solution of the entire line agree for certain line configurations. The physical and geometrical parameters for these corresponding configurations are determined in this paper.
\end{abstract}

\section{Introduction}

Transmission-Line Super Theory (TLST) was introduced by Haase and Nitsch $(2001,2003)$ more than one decade ago. In this theory Maxwell's equations are represented for a system of lossless nonuniform thin transmission lines in a system of equations which have the same structure as the telegrapher equations. In particular, the TLST equations take into account all field modes and physical effects that might occur, including radiation losses. It surpasses the classical transmission-line theory, which is a special case. Their complex parameters are local and frequency dependent and are obtained by the solution of integral equations.
In the paper a practical classical transmission-line (cTL) is regarded. The considered TL consists of a finite part parallel to the ground plane and two vertical risers connecting the horizontal part to the conducting ground plane at the ends.

In Sect. 2 the classical analysis of the TL is briefly described and the classical reflection coefficients are introduced. In Sect. 3 the fundamentals of TLST are presented and the finite transmission line with risers is analyzed using the numerical TLST procedure. The local and frequency dependent parameter matrix elements representing the per unit length inductance and capacitance values are discussed. A procedure is shown where the whole TL can be separated in uniform and nonuniform parts. Only for the nonuniform parts TLST has to be used and the asymptotic part can be handled classically. In Sect. 4 novel local and frequency dependent reflection coefficients are introduced and it is shown how they are related to the matrizant elements of the TLST. Finally it is shown how the current on the TL can be calculated using the reflection coefficients. Numerical results for the finite uniform TL with risers are shown and discussed in Sect. 5. Calculated results for the novel reflection coefficients and current values on the TL are shown for several geometrical constitutions of the TL. Finally the results are discussed and summarized in Sect. 6.

\section{A finite TL in classical transmission-line theory}

Classical transmission-line theory (cTLT) does not take care of effects of finite open ends or of risers to the ground plane. The TL is regarded physically infinite and mathematically a total length is designated to meet the right resonance frequencies. The dominating TEM mode of such a TL is commonly 


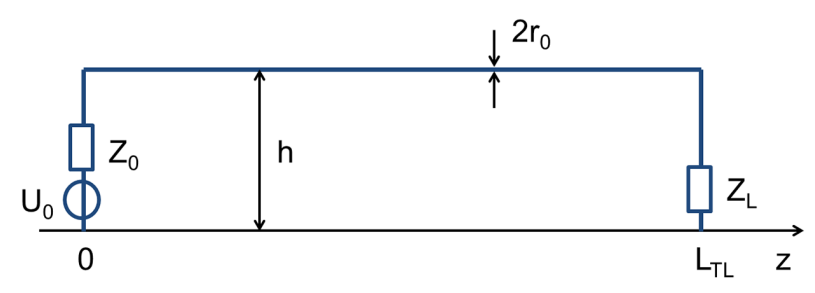

Figure 1. Geometry of a horizontal finite TL (length $L_{\mathrm{TL}}$ ) with height $h$ over PEC ground including two risers.

described by the classical transmission-line equations

$$
\begin{aligned}
& \frac{d U(z)}{d z}+j \omega L_{\mathrm{cTL}}^{\prime} I(z)=0 \\
& \frac{d I(z)}{d z}+j \omega C_{\mathrm{cTL}}^{\prime} U(z)=0,
\end{aligned}
$$

where $z$ is the axial orientation of the TL (see Fig. 1) and $U(z)$ and $I(z)$ are the complex voltage and current distributions on the line. The classical (and constant) per unit length (p.u.l) inductance and capacitance are named as $L_{\mathrm{cTL}}^{\prime}$ and $C_{\text {cTL }}^{\prime}$, respectively.

The finite classical TL with risers over a conducting ground plane (PEC) regarded in this work is shown in Fig. 1. To simplify the presentation the following parameters for the TL are chosen, although the outlined method works in general: wire radius $r_{0}=0.5 \mathrm{~mm}$; height over ground $h=5 \mathrm{~cm}$; length of the horizontal part $L_{\mathrm{TL}}=2 \mathrm{~m}$; total arc length of the TL $L=L_{\mathrm{TL}}+2 h=2.1 \mathrm{~m}$. At the beginning the line is fed by a lumped source with voltage $U_{0}=1 \mathrm{~V}$ and source impedance $Z_{0}=50 \Omega$. The line is terminated with a load impedance $Z_{\mathrm{L}}=50 \Omega$. The formulas for the classical p.u.l. inductance $L_{\mathrm{cTL}}^{\prime}$ and capacitance $C_{\mathrm{cTL}}^{\prime}$ are

$$
\begin{aligned}
& L_{\mathrm{cTL}}^{\prime}=\frac{\mu_{0}}{2 \pi} \ln \left(\frac{2 h}{r_{0}}\right)=1.06 \times 10^{-6} \frac{\mathrm{V} \mathrm{s}}{\mathrm{Am}} \\
& C_{\mathrm{cTL}}^{\prime}=\frac{2 \pi \epsilon_{0}}{\ln \left(\frac{2 h}{r_{0}}\right)}=1.05 \times 10^{-11} \frac{\mathrm{As}}{\mathrm{Vm}},
\end{aligned}
$$

resulting in a characteristic line impedance of $Z_{\mathrm{C}}=$ $\sqrt{L_{\mathrm{cTL}}^{\prime} C^{\prime}{ }_{\mathrm{cTL}}^{-1}}=318 \Omega$.

A current wave originating at $+\infty$, traveling on the horizontal part of the TL in $-z$ direction and being reflected at the beginning of the TL at $z=0$ can be expressed using the classical left-hand current reflection coefficient $R_{+}^{\text {class }}$ as

$I(z)=I_{1}\left(e^{j k z}+R_{+}^{\text {class }} e^{-j k z}\right)$.

with $I_{1}$ being an appropriate constant. From Eq. (1) the expression for the voltage $U(z)=-\frac{1}{j \omega C^{\prime}} \frac{d I(z)}{d z}$ can be deduced and together with Eq. (4) the result for the left-hand current reflection coefficient is calculated as

$$
R_{+}^{\text {class }}=e^{2 j k z} \frac{Z_{\mathrm{C}} I(z)+U(z)}{Z_{\mathrm{C}} I(z)-U(z)}=\frac{Z_{\mathrm{C}}-Z_{0}}{Z_{\mathrm{C}}+Z_{0}} .
$$

For the final step in Eq. (5) the general classical solutions for $U(z)$ and $I(z)$ for a uniform TL

$$
\left(\begin{array}{c}
U(z) \\
I(z)
\end{array}\right)=\left(\begin{array}{cc}
\cos (k z) & -j Z_{\mathrm{C}} \sin (k z) \\
-\frac{j}{Z_{\mathrm{C}}} \sin (k z) & \cos (k z)
\end{array}\right) \cdot\left(\begin{array}{c}
U(0) \\
I(0)
\end{array}\right),
$$

was used. It is obvious that in cTLT the reflection coefficients are constant.

Corresponding considerations using a current wave traveling on the horizontal part of the line originating at $-\infty$ lead to the (constant) classical right-hand current reflection coefficient $R_{-}^{\text {class }}$ as

$$
R_{-}^{\text {class }}=e^{-2 j k\left(z-L_{\mathrm{TL}}\right)} \frac{Z_{\mathrm{C}} I(z)-U(z)}{Z_{\mathrm{C}} I(z)+U(z)}=\frac{Z_{\mathrm{C}}-Z_{\mathrm{L}}}{Z_{\mathrm{C}}+Z_{\mathrm{L}}} .
$$

\section{TLST analysis of a finite TL with risers}

\subsection{Fundamentals of TLST}

Transmission-line super theory (Haase and Nitsch, 2001; Haase et al., 2003; Haase, 2005; Nitsch et al., 2009; Nitsch and Tkachenko, 2010) is a full wave description of Maxwell's equations cast into the form of telegrapher's equations. For a single wire system (with return conductor or ground plane) the super theory transmission-line equation for lumped sources or loads at the line ends in the potentialcurrent representation states (Rambousky et al., 2012)

$$
\frac{\partial}{\partial l}\left[\begin{array}{c}
\varphi(l, f) \\
i(l, f)
\end{array}\right]+j \omega \overline{\mathbf{P}}^{*(1)}(l, f)\left[\begin{array}{c}
\varphi(l, f) \\
i(l, f)
\end{array}\right]=\left[\begin{array}{l}
0 \\
0
\end{array}\right] .
$$

The potential on the transmission-line is denoted by $\varphi(l, f)$ and the current by $i(l, f)$. The best choice for the line parameter is the (natural) arc length $l$ of the line and $f$ is the frequency. The super matrix $\overline{\mathbf{P}}^{*(1)}$ is the transmission-line parameter matrix. In the case of a one wire system $\overline{\mathbf{P}}^{*(1)}$ is a 2 by 2 matrix. In contrast to cTLT the transmission-line parameter matrix $\overline{\mathbf{P}}^{*(1)}(l, f)$ now is complex valued and both local $(l)$ and frequency $(f)$ dependent. This parameter matrix is calculated by an iteration process starting with a low frequency approximation in the zeroth iteration step resulting in a frequency independent but already local parameter matrix $\overline{\mathbf{P}}^{*(0)}(l)$ (Nitsch et al., 2009; Rambousky et al., 2012). In previous work we could show that already the first iteration step results in an acceptable accuracy (Rambousky et al., 2013a). The general solution of the super theory transmission-line equation (8) for the one wire case can be written as

$$
\left[\begin{array}{c}
\varphi(l, f) \\
i(l, f)
\end{array}\right]=\mathcal{M}_{l_{0}}^{l}\left\{-j \omega \overline{\mathbf{P}}^{*(1)}\right\}\left[\begin{array}{l}
\varphi\left(l_{0}, f\right) \\
i\left(l_{0}, f\right)
\end{array}\right],
$$

where the expression $\mathcal{M}_{l_{0}}^{l}$ is the so called matrizant or product integral (Gantmacher, 1984), and $l_{0}$ and $l>l_{0}$ represent two spatial positions on the TL. Regarding only lumped 


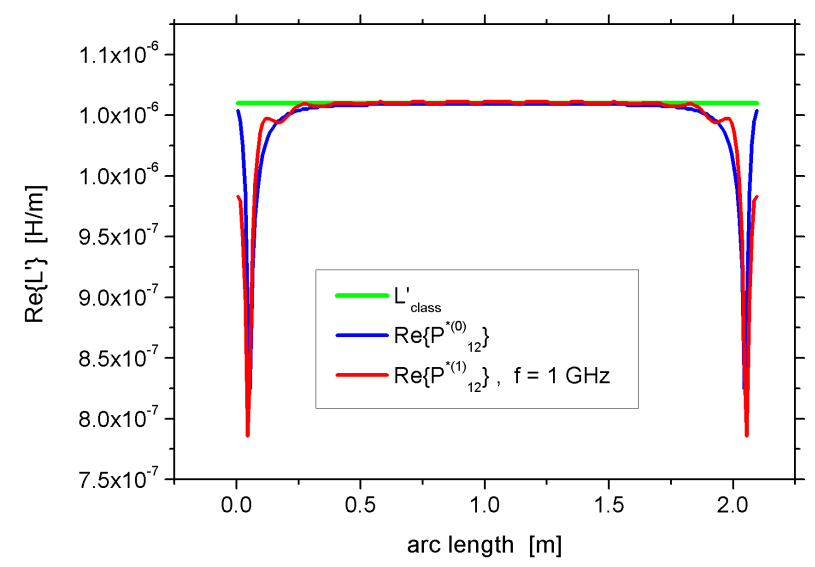

Figure 2. Parameter matrix elements representing the real part of the p.u.l. inductance for TLST analysis of the TL configuration.

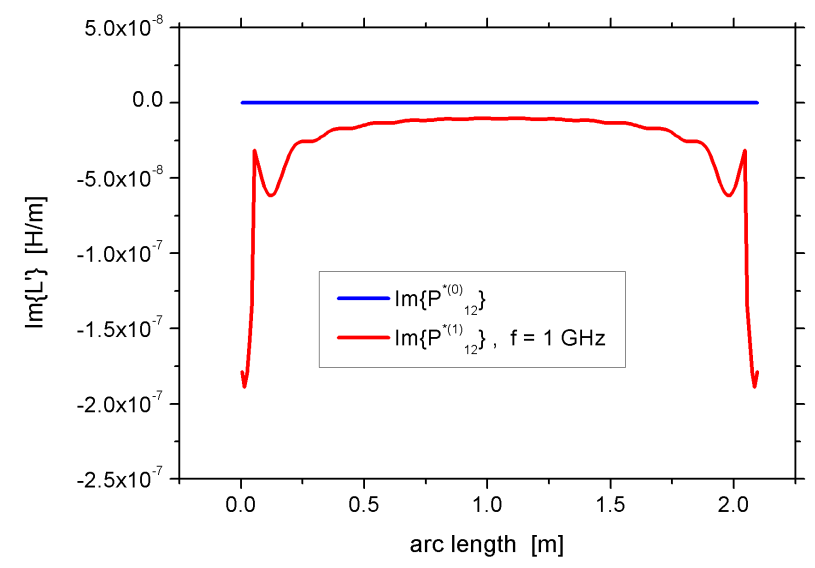

Figure 3. Parameter matrix elements representing the imaginary part of the p.u.l inductance for TLST analysis of the TL configuration.

sources or lumped loads at the ends of the wires, Eq. (9) can be calculated using the appropriate boundary conditions of the TL model.

\subsection{TLST parameter elements for a finite TL with risers}

The parameter matrices $\overline{\mathbf{P}}^{*(0)}(l)$ and $\overline{\mathbf{P}}^{*(1)}(l, f)$ resulting from the TLST iteration process are independent of the lumped sources and loads. The $\mathbf{P}_{12}^{*}$ elements representing the p.u.l. inductance are shown in Fig. 2 (real part) and in Fig. 3 (imaginary part).

Figure 2 indicates that in the TLST the classical p.u.l. inductance $L_{\mathrm{cTL}}^{\prime}$ is reached at a certain distance away from the risers for the configuration presented in Fig. 1. The graph of the inductance bends when approaching the ends of the horizontal part of the line and reaches its minima at the ends of the horizontal parts. A reason for this behavior can be found in Nitsch et al. (2009). When passing through the risers it in-

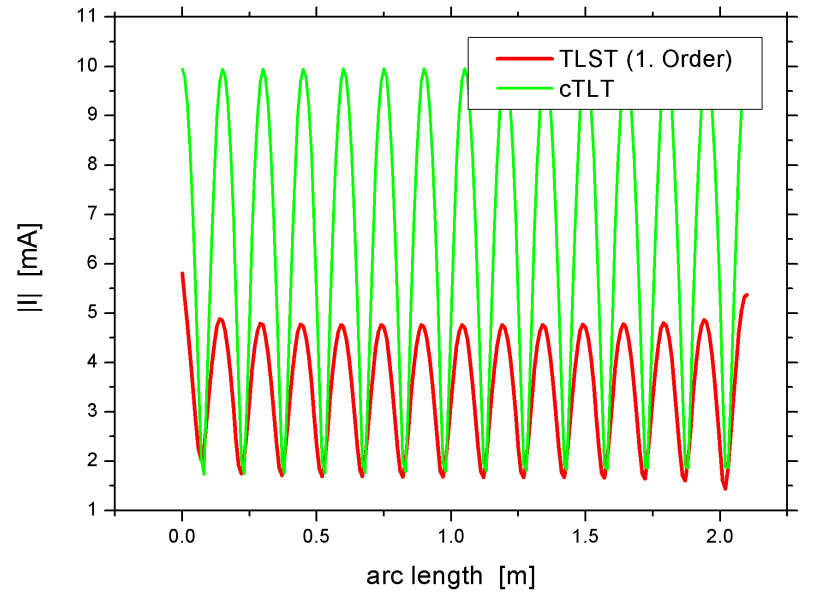

Figure 4. Current on the TL calculated using TLST approach for $f=1 \mathrm{GHz}$ compared to classical TL theory.

creases again. Also the imaginary part of $L^{\prime}$ deviates significantly from the classical value (zero) at the risers, indicating the most radiative parts of the TL.

The current on the TL calculated using TLST with first order iteration parameter matrix is shown in Fig. 4 for a frequency of $1 \mathrm{GHz}$. To have a comparable arc length, the total length of the classical TL was also set to $2.1 \mathrm{~m}$. It is clearly seen that the real current distribution deviates significantly from the classical theory, mainly because of the radiating losses at the used frequency.

\subsection{Decomposition of the TL based on the group property of the matrizant}

In the example of Fig. 1 the TL can be decomposed in the left-hand riser part, the uniform middle part (asymptotic region) and the right-hand riser part. Attention has to be paid that the junctions are located where the composed TL shows almost classical behavior (see Fig. 2). Therefore the junctions have to be sufficiently far away from the riser, like at $z_{1}$ and $z_{2}$ as shown in Fig. 5. Now, the TLST parameter matrices for the single parts of the decomposed TL can be calculated. Because even the tail end of an otherwise classical TL shows significant deviation of the line parameters in TLST, the elements of the parameter matrix have to be adjusted due to the junction. For the used TL with risers the asymptotic region (part II) was defined as a classical TL with constant line parameters using Eqs. (2) and (3). The riser parts I and III were adjusted for the junctions by hand to ignore the tail ends and to meet the classical values at the junctions. This is shown in Fig. 6 with the dashed curves.

For the whole TL current and potential in the load $Z_{\mathrm{L}}$ at the end of the line $(l=L)$ can be calculated using the first 


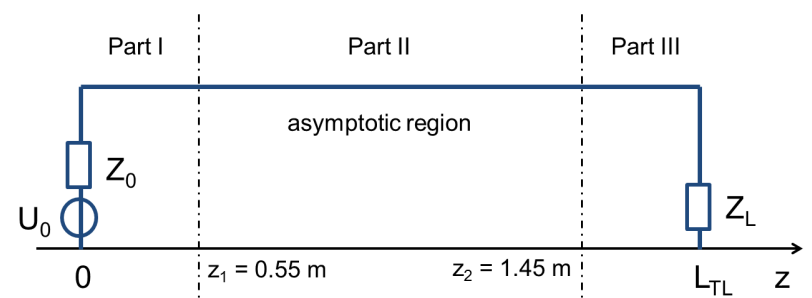

Figure 5. Partitioning of the nonuniform TL of Fig. 1 into an asymptotic region (part II) and the two riser regions (parts I and III).

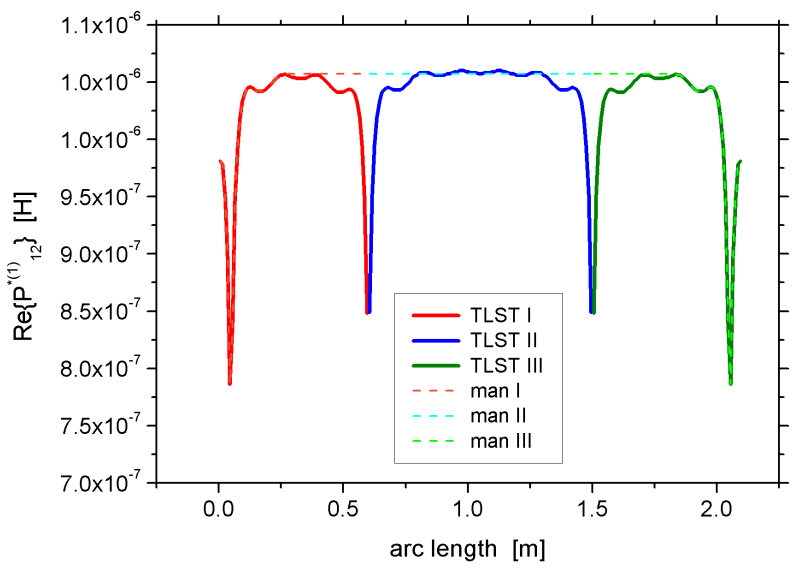

Figure 6. Real part of the parameter matrix element $\bar{P}_{12}^{*(1)}$ for the defined three parts of the nonuniform TL and their manual adjustment at the junctions.

order parameter matrix $\overline{\mathbf{P}}^{*(1)}$

$\left[\begin{array}{c}\varphi(L) \\ i(L)\end{array}\right]=\mathcal{M}_{0}^{L}\left\{-j \omega \overline{\mathbf{P}}^{*(1)}\right\}\left[\begin{array}{c}\varphi(0) \\ i(0)\end{array}\right]=\mathbf{M}_{\mathrm{L}}\left[\begin{array}{c}\varphi(0) \\ i(0)\end{array}\right]$.

The matrix $\mathbf{M}_{\mathrm{L}}$ is the matrizant over the whole arc length of the TL using the parameter matrix $\overline{\mathbf{P}}^{*(1)}$ of the whole TL.

On the other hand, current and potential in the load $Z_{\mathrm{L}}$ can be calculated using the matrizants $\mathbf{M}_{\mathrm{I}}^{\mathrm{man}}, \mathbf{M}_{\mathrm{II}}^{\mathrm{man}}$ and $\mathbf{M}_{\mathrm{III}}^{\operatorname{man}}$ of the single parts I, II and III of the TL with the manually (at the junctions) adapted parameter matrices as

$\left[\begin{array}{c}\varphi(L) \\ i(L)\end{array}\right]=\mathbf{M}_{\mathrm{III}}^{\operatorname{man}} \cdot \mathbf{M}_{\mathrm{II}}^{\operatorname{man}} \cdot \mathbf{M}_{\mathrm{I}}^{\operatorname{man}}\left[\begin{array}{l}\varphi(0) \\ i(0)\end{array}\right]$.

For example $\mathbf{M}_{\mathrm{I}}^{\operatorname{man}}$ is the matrizant covering the arc length from $l=0$ to $l=z_{1}+h=0.6 \mathrm{~m}$ using the manually (at the junction) adapted first order parameter matrix $\overline{\mathbf{P}}_{\mathrm{I}, \text { man }}^{*(1)}$ resulting in

$\mathbf{M}_{\mathrm{I}}^{\text {man }}=\mathcal{M}_{0}^{z_{1}+h}\left\{-j \omega \overline{\mathbf{P}}_{\mathrm{I}, \text { man }}^{*(1)}\right\}$.

To validate the equivalence of Eqs. (10) and (11) the current in $Z_{\mathrm{L}}$ at the end of the TL was calculated in both ways. The result is shown in Fig. 7 and gives very good agreement. It

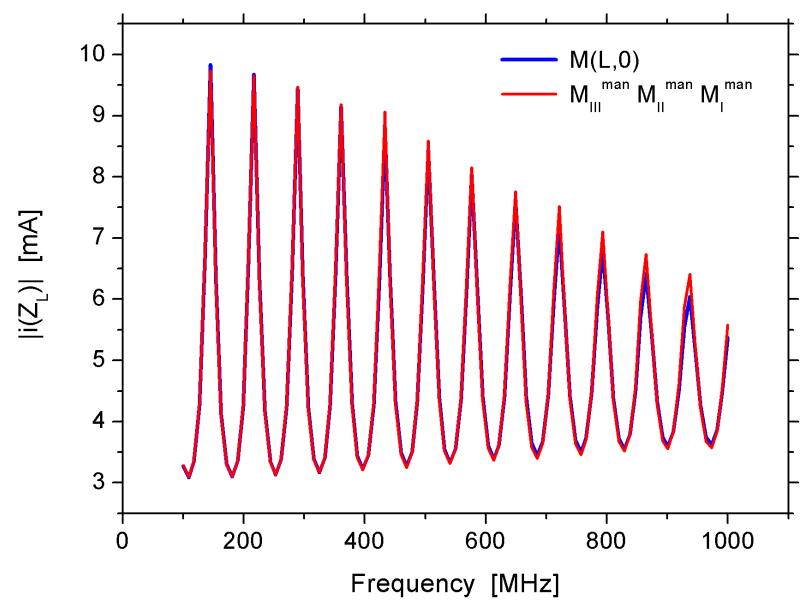

Figure 7. Current in the load $Z_{\mathrm{L}}$ at the end of the TL of Fig. 1.

has to be mentioned again that in the assembled solution the middle part (part II) of the TL was regarded as a pure classical TL. The results so far show that the current distribution on a real TL with risers can be calculated by dividing the line in uniform and nonuniform parts. The nonuniform parts have to be calculated using an advanced TLT, like TLST. The uniform parts can be handled as classical TL. The overall matrizant of the TL can be assembled by multiplying the single matrizants of the TL parts in correct order.

\section{Novel local and frequency dependent current reflection coefficients and amplitude functions}

The idea now is to transfer the concept of current reflection coefficients from cTLT to a realistic finite transmission-line with risers at both ends.

\subsection{Derivation of the novel current reflection coefficients using TLST}

In TLST voltage $U(z)$ is replaced by the potential $\varphi(l)$ and current $I(z)$ by $i(l)$. Again $l$ is the natural parameter of the TL (arc length) including the risers. As an extension of Eqs. (5) and (7) the now $l$ and frequency dependent current reflection coefficients can also be defined as the quotient of an incoming and outgoing current wave as

$\widetilde{R}_{+}(l):=e^{2 j k l} \frac{Z_{\mathrm{C}} i(l)+\varphi(l)}{Z_{\mathrm{C}} i(l)-\varphi(l)}$

and

$\widetilde{R}_{-}(l):=e^{-2 j k(l-L)} \frac{Z_{\mathrm{C}} i(l)-\varphi(l)}{Z_{\mathrm{C}} i(l)+\varphi(l)}$.

The advanced current reflection coefficients $\widetilde{R}_{+}(l)$ and $\widetilde{R}_{-}(l)$ now are expressed using the results of TLST calculations. Due to the group feature of the resulting matrizants (see 




Figure 8. Transmission-line configuration for derivation of the right-hand current reflection coefficient $\widetilde{R}_{-}(l)$ of the TL with risers.

Sect. 3.3), the matrizant of the whole TL can be composed as a matrix product of matrizants representing parts of the TL. Therefore, actually only the riser parts I and III have to be calculated using TLST and the classical matrizant can be used for the asymptotic region (part II). A theoretical restriction for our approach is that no radiation coupling between the two riser parts of the TL is allowed. This is assured if the horizontal length $L_{\mathrm{TL}}$ of the TL is large compared to the height $h$ over ground.

\subsubsection{The right-hand reflection coefficient $\tilde{R}_{-}(l)$}

In Fig. 8 the TL configuration for derivation of the right-hand current reflection coefficient $R_{-}(l)$ is depicted. Imagine a current wave coming from $-\infty$ travels in positive $z$ direction, gets reflected at the right-hand riser and travels back to $-\infty$. The classical telegrapher's equations are valid in the asymptotic region, defined by $L_{1} \leq l \leq L_{2}$. The matrizant $\mathcal{M}_{\mathrm{L}}^{l}\left\{-j \omega \overline{\mathbf{P}}^{*(1)}\right\} \equiv \mathcal{M}(l, L)$ can be decomposed in

$\mathcal{M}(l, L)=\mathcal{M}\left(l, L_{2}\right) \cdot \mathcal{M}\left(L_{2}, L\right)$,

where the second factor on the right side of Eq. (15) is independent of $l$. The $l$ dependence is restricted to the asymptotic region. For $L_{\mathrm{TL}} \gg h$ the radiation coupling is negligible and $\widetilde{R}_{-}(l)$ is independent of $\widetilde{R}_{+}(l)$.

In a next step the quotient in Eq. (14) has to be expressed by matrizants of the TLST calculation. Generally the relation

$\left[\begin{array}{c}\varphi\left(l_{2}\right) \\ i\left(l_{2}\right)\end{array}\right]=\mathcal{M}\left(l_{2}, l_{1}\right)\left[\begin{array}{c}\varphi\left(l_{1}\right) \\ i\left(l_{1}\right)\end{array}\right] \quad \forall l_{1}, l_{2} \in[0, L]$

holds. Setting $l_{2}=l$ and $l_{1}=L$ and using the boundary condition $\varphi(L)=U_{\mathrm{L}}=Z_{\mathrm{L}} i(L)$ the potential-current vector at arc length $l$ can be expressed as

$$
\begin{aligned}
& {\left[\begin{array}{c}
\varphi(l) \\
i(l)
\end{array}\right]=\mathcal{M}(l, L)\left[\begin{array}{c}
\varphi(L) \\
i(L)
\end{array}\right]} \\
& =i(L)\left[\begin{array}{ll}
\mathcal{M}_{11}(l, L) & \mathcal{M}_{12}(l, L) \\
\mathcal{M}_{21}(l, L) & \mathcal{M}_{22}(l, L)
\end{array}\right] \cdot\left[\begin{array}{c}
Z_{\mathrm{L}} \\
1
\end{array}\right] .
\end{aligned}
$$

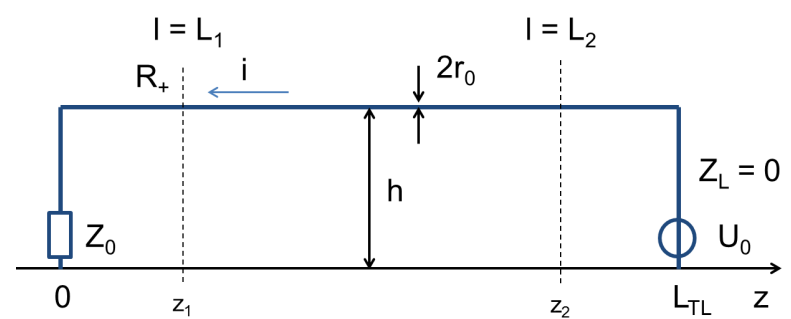

Figure 9. Transmission-line configuration for derivation of the lefthand current reflection coefficient $\widetilde{R}_{+}(l)$ of the TL with risers.

Inserting the results for $\varphi(l)$ and $i(l)$ in Eq. (14) leads to the advanced expression for the right-hand current reflection coefficient $\widetilde{R}_{-}(l)$ which is now local and frequency dependent.

$$
\begin{aligned}
& \widetilde{R}_{-}(l)=e^{-2 j k(l-L)} \\
& \cdot\left(Z_{\mathrm{L}}\left[-\mathcal{M}_{11}(l, L)+Z_{\mathrm{C}} \mathcal{M}_{21}(l, L)\right]\right. \\
& \left.+\left(-\mathcal{M}_{12}(l, L)+Z_{\mathrm{C}} \mathcal{M}_{22}(l, L)\right)\right) \\
& \cdot\left(Z_{\mathrm{L}}\left[\mathcal{M}_{11}(l, L)+Z_{\mathrm{C}} \mathcal{M}_{21}(l, L)\right]\right. \\
& \left.+\mathcal{M}_{12}(l, L)+Z_{\mathrm{C}} \mathcal{M}_{22}(l, L)\right)^{-1} .
\end{aligned}
$$

\subsubsection{The left-hand reflection coefficient $\widetilde{\boldsymbol{R}}_{+}(l)$}

The TL configuration for the derivation of $\widetilde{R}_{+}(l)$ is depicted in Fig. 9. It is assumed that a current wave traveling in $-z$ direction gets reflected at the left-hand side of the TL. Using the same concept as before the matrizant can be decomposed in a non $l$ dependent part and an $l$ dependent part (asymptotic region), that is $\mathcal{M}(l, 0)=\mathcal{M}\left(l, L_{1}\right) \cdot \mathcal{M}\left(L_{1}, 0\right)$. The second one includes the essential physical property of the reflection process.

Using the same derivation method as before (now setting $l_{1}=0$ and $l_{2}=l$ ) one gets the following advanced expression for the left-hand current reflection coefficient $\widetilde{R}_{+}(l)$ which is again local and frequency dependent.

$$
\begin{aligned}
& \widetilde{R}_{+}(l)=e^{2 j k l} \\
& \cdot\left(-Z_{0}\left[\mathcal{M}_{11}(l, 0)+Z_{\mathrm{C}} \mathcal{M}_{21}(l, 0)\right]\right. \\
& \left.+\mathcal{M}_{12}(l, 0)+Z_{\mathrm{C}} \mathcal{M}_{22}(l, 0)\right) \\
& \cdot\left(-Z_{0}\left[-\mathcal{M}_{11}(l, 0)+Z_{\mathrm{C}} \mathcal{M}_{21}(l, 0)\right]\right. \\
& \left.-\mathcal{M}_{12}(l, 0)+Z_{\mathrm{C}} \mathcal{M}_{22}(l, 0)\right)^{-1} .
\end{aligned}
$$

\subsection{Derivation of the amplitude function $\widetilde{C}_{+}(l)$}

The configuration for calculating the amplitude function of a forward $(+z$-direction) traveling current wave is depicted in Fig. 5. It is assumed that the load impedance is ideal $\left(Z_{\mathrm{L}}=\right.$ $Z_{C}$ ) for all used frequencies and the traveling current wave is not reflected at the end of the TL. With $i(l)=\widetilde{C}_{+}(l) e^{-j k l}$ and $\varphi(l)=Z_{\mathrm{C}} \widetilde{C}_{+}(l) e^{-j k l}$ the following expression for $\widetilde{C}_{+}(l)$ can be derived by summation:

$\widetilde{C}_{+}(l)=e^{j k l} \frac{i(l) Z_{\mathrm{C}}+\varphi(l)}{2 Z_{\mathrm{C}}}$. 


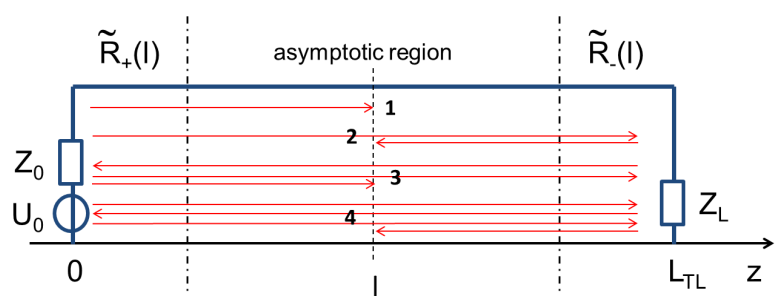

Figure 10. Transmission-line configuration for derivation of the left-hand amplitude function $\widetilde{C}_{+}(l)$ and the description of multiple reflections.

The quotient in Eq. (20) can be expressed again using the matrizants of the TLST calculation. With the relation

$\left[\begin{array}{c}\varphi(l) \\ i(l)\end{array}\right]=\mathcal{M}(l, 0)\left[\begin{array}{c}U_{0}-Z_{0} i(0) \\ i(0)\end{array}\right]$,

leading to the expressions

$\varphi(l)=i(l) Z_{\mathrm{C}}$

$=\mathcal{M}_{11}(l, 0)\left(U_{0}-Z_{0} i(0)\right)+\mathcal{M}_{12}(l, 0) i(0)$

$i(l)=\mathcal{M}_{21}(l, 0)\left(U_{0}-Z_{0} i(0)\right)+\mathcal{M}_{22}(l, 0) i(0)$,

a result for the characteristic impedance $Z_{\mathrm{C}}$ can be received by division:

$Z_{\mathrm{C}}=\frac{\mathcal{M}_{11}(l, 0)\left[U_{0}-Z_{0} i(0)\right]+\mathcal{M}_{12}(l, 0) i(0)}{\mathcal{M}_{21}(l, 0)\left[U_{0}-Z_{0} i(0)\right]+\mathcal{M}_{22}(l, 0) i(0)}$.

Solving Eq. (24) for $i(0)$ yields

$$
\begin{aligned}
& i(0)=U_{0}\left[\mathcal{M}_{21}(l, 0) Z_{\mathrm{C}}-\mathcal{M}_{11}(l, 0)\right]\left[Z_{0} Z_{\mathrm{C}} \mathcal{M}_{21}(l, 0)\right. \\
& \left.-\mathcal{M}_{22}(l, 0) Z_{\mathrm{C}}-\mathcal{M}_{11}(l, 0) Z_{0}+\mathcal{M}_{12}(l, 0)\right]^{-1} .
\end{aligned}
$$

The intermediate result Eq. (25) has to be insertet into Eqs. (22) and (23). Then using Eq. (20) and considering that the determinant of $\mathcal{M}(l, 0)$ is always 1 , results in the final expression for $\widetilde{C}_{+}(l)$ :

$\widetilde{C}_{+}(l)=U_{0} e^{j k l}\left[-Z_{0} Z_{\mathrm{C}} \mathcal{M}_{21}(l, 0)\right.$

$\left.+\mathcal{M}_{22}(l, 0) Z_{\mathrm{C}}+\mathcal{M}_{11}(l, 0) Z_{0}-\mathcal{M}_{12}(l, 0)\right]^{-1}$

Inserting the classical matrix elements from Eqs. (6) into (26) the cTLT expression for the amplitude function, $\widetilde{C}_{+}^{\text {class }}=$ $U_{0} /\left(Z_{0}+Z_{\mathrm{C}}\right)$, is received.

\subsection{Calculation of the TL current using novel reflection coefficients and amplitude function}

In the last step the current on the TL has to be determined using the previously derived reflection coefficients $\widetilde{R}_{-}(l)$, $\widetilde{R}_{+}(l)$ and the amplitude function $\widetilde{C}_{+}(l)$. Therefore the configuration of Fig. 10 is used with arbitrary loads $Z_{0}$ and $Z_{\mathrm{L}}$. A forward traveling outgoing current wave $i_{1}(l)=\widetilde{C}_{+}(l) e^{-j k l}$ would be reflected at the end and the current wave $i_{2}(l)=$

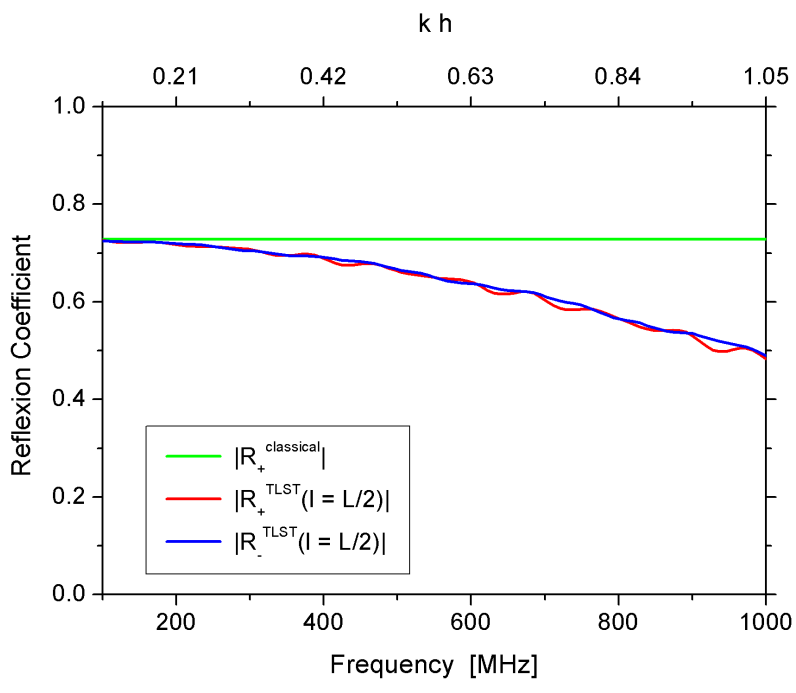

Figure 11. Reflection coefficients $\left|\widetilde{R}_{+}\right|,\left|\widetilde{R}_{-}\right|$at the center point for the classical TL with risers of Fig. 1

$\widetilde{C}_{+}(l) e^{-j k l} \widetilde{R}_{-} e^{-j k(L-l)}$ would travel back. This reflected wave would be reflected again at the beginning of the TL and the current wave $i_{3}(l)=\widetilde{C}_{+}(l) e^{-j k l} \widetilde{R}_{-} e^{-j k(L-l)} \widetilde{R}_{+} e^{-j k l}$ would travel also again to the end of the TL. Theoretically this procedure would be repeated endlessly leading to an expression for the current wave with two infinite sums

$i(l)=\widetilde{C}_{+}(l) \sum_{n=0}^{\infty}\left(e^{-j k L} \widetilde{R}_{-} e^{-j k L} \widetilde{R}_{+}\right)^{n} e^{-j k l}$
$+\widetilde{C}_{+}(l) e^{-j k L} \widetilde{R}_{-} \sum_{n=0}^{\infty}\left(e^{-j k L} \widetilde{R}_{+} e^{-j k L} \widetilde{R}_{-}\right)^{n} e^{-j k(L-l)}$.

The two sums in Eq. (27) represent geometrical series and can be simplified leading to the final result for the current on the TL

$i(l)=\frac{\widetilde{C}_{+}(l)\left(e^{-j k l}+\widetilde{R}_{-} e^{-2 j k L} e^{j k l}\right)}{1-\widetilde{R}_{-} \widetilde{R}_{+} e^{-2 j k L}}$.

It has to be mentioned that for the asymptotic region, that is $l \in\left[L_{1}, L_{2}\right]$, the reflection coefficients are constant.

When the current on the TL is known for example from a full wave simulation the current reflection coefficients can be calculated. This is shown for $\widetilde{R}_{+}(l)$. For the asymptotic region a backward traveling wave can be expressed as $i(l)=$ $\widetilde{I}_{1}\left(e^{j k l}+\widetilde{R}_{+} e^{-j k l}\right)$. A straight forward calculation results in

$\widetilde{R}_{+}(l)=\left(\frac{j k i(l)-\frac{d i(l)}{d l}}{j k i(l)+\frac{d i(l)}{d l}}\right) e^{2 j k l}$.

\section{Numerical calculations for classical TL with risers}

First, the TL from Fig. 1 is regarded with the before mentioned line parameters $L_{\mathrm{TL}}=2 \mathrm{~m}, L=2.1 \mathrm{~m}, h=5 \mathrm{~cm}$ and 




Figure 12. Current for different positions on the TL calculated using the reflection coefficients.

$r_{0}=0.5 \mathrm{~mm}$. The TL is driven by a voltage source $U_{0}=1 \mathrm{~V}$ with source impedance $Z_{0}=50 \Omega$ and terminated by a load $Z_{\mathrm{L}}=50 \Omega$. Elements of the parameter matrix $\overline{\mathbf{P}}^{*(1)}$ (TLST) are shown in Figs. 2 and 3. Using formulas Eqs. (19) and (18) the current reflection coefficients $\left|\widetilde{R}_{+}\right|$and $\left|\widetilde{R}_{-}\right|$were calculated for the center position on the TL at $l=L / 2$ in the frequency range from $100 \mathrm{MHz}$ to $1 \mathrm{GHz}$ as shown in Fig. 11. It is clearly seen that the current reflection coefficients deviate from their classical value significantly with rising frequency because of the radiated energy losses. Because source and load impedance are the same in this configuration, $\left|\widetilde{R}_{+}\right|$and $\left|\widetilde{R}_{-}\right|$have the same value.

Using Eq. (28) the current can be calculated for different positions and frequencies. Fig. 12 shows the results for the positions $L_{1}, L / 2$ and $L_{2}$. Also shown (black dash-dotted line) is the current $|I(L / 2)|$ resulting from a MoM calculation using the Concept-II code (Brüns et al., 2011). The correspondence between full wave analysis and calculation using novel local and frequency dependent reflection coefficients is excellent.

In Fig. 13 the reflection coefficients $\left|\widetilde{R}_{-}\right|$are shown for the TL from Fig. 1 with $h=5 \mathrm{~cm}$ for different loads $Z_{\mathrm{L}}$ (short circuited, $50 \Omega$, matched and open). The source impedance remains at $Z_{0}=50 \Omega$, so $\left|\widetilde{R}_{+}\right|$is the same for all $Z_{\mathrm{L}}$ values and is explicitly shown for the open case. The classical current reflection coefficient for an open TL is negative because of the necessary phase shift of the current wave. In Fig. 13 the absolute value of $\widetilde{R}_{-}$is presented, but of course for $\omega \rightarrow 0$ the real part of $\widetilde{R}_{-}$would tend to the value -1 . For a matched load the classical reflection coefficient is zero. That means the current wave would completely be absorbed in the load and no reflected wave would be produced. In a real TL with risers there is no fixed matched load for all frequencies any longer (Rambousky et al., 2013b). The nonuniformity of a

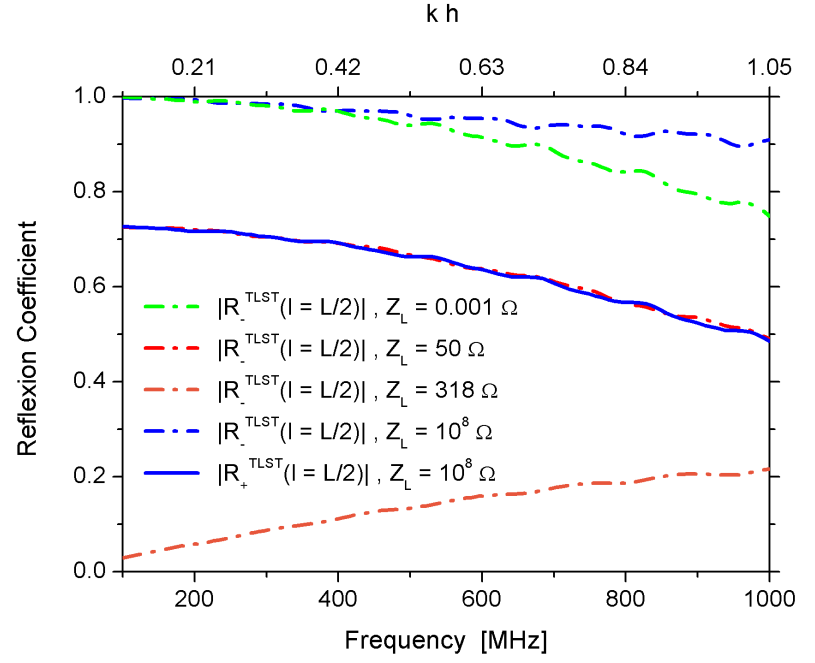

Figure 13. Reflection coefficients at $l=L / 2$ for different load impedances $Z_{\mathrm{L}}$.

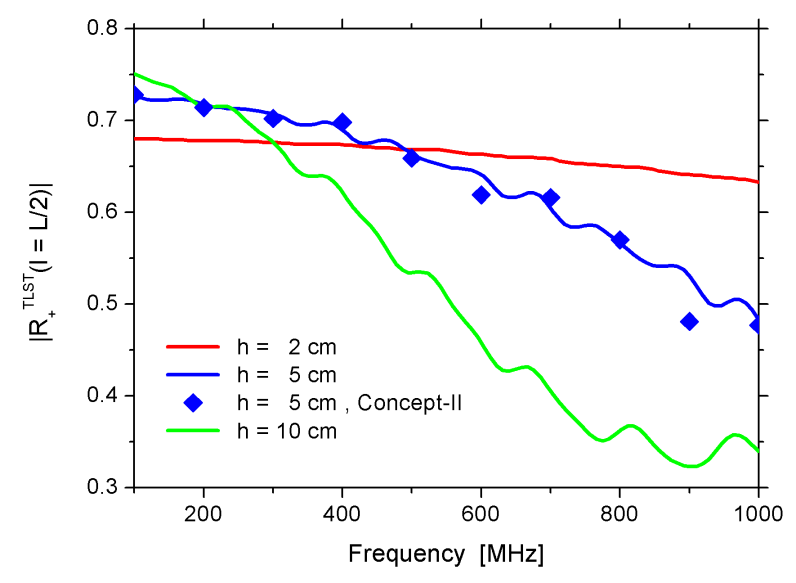

Figure 14. Reflection coefficients at $l=L / 2$ for different heights $h$ of the TL over PEC ground.

real TL is responsible for the scattering of the current wave at the local (e.g. bends) or distributed (e.g. varying height over ground) scattering centers. With a classical matched load impedance the current reflection coefficient $\left|\widetilde{R}_{-}\right|$rises with frequency as can clearly be seen in Fig. 13.

Another interesting fact is the influence of the height $h$ of the TL over ground on the reflection coefficient. With decreasing height $h$ a TL should show increasingly classical behavior. This can be seen also in the gradient of the reflection coefficients. In Fig. 14 it is shown that for decreasing height $h$ the frequency dependence of $\left|\widetilde{R}_{+}\right|$decreases also and would approach the constant classical value for $h \rightarrow 0$. The curve with the blue diamonds in Fig. 14 was produced by calculating the current on the TL with the MoM code Concept-II and using Eq. (29) to calculate $\widetilde{R}_{+}(l)$. There is again a good correspondence between MoM and TLST results. 


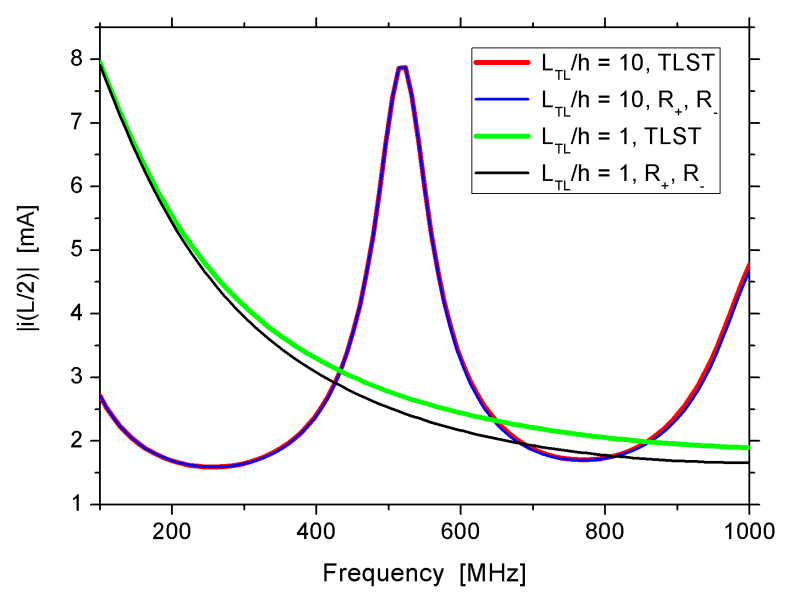

Figure 15. Current at the middle of the TL $(l=L / 2)$ for a constant height $h=5 \mathrm{~cm}$ and different horizontal length $L_{\mathrm{TL}}$.

As mentioned before the original TLST calculation of the current on the TL is an exact solution while the calculation using the novel reflection coefficients is still an approximation because the mutual influence of the risers is neglected. When the ratio of the horizontal part of the TL and the height above the conducting ground plane, $L_{\mathrm{TL}} / h$, is large enough there will be no significant influence due to the risers. The current on the TL then should be the same as for a pure TLST calculation and a current calculation using the above reflection coefficient method at least for the asymptotic region. This can be seen in Fig. 15 for the ratio $L_{\mathrm{TL}} / h=50 \mathrm{~cm} / 5 \mathrm{~cm}=10$. The correspondence is nearly perfect. Reducing the ratio dramatically to $L_{\mathrm{TL}} / h=$ $5 \mathrm{~cm} / 5 \mathrm{~cm}=1$ where the length of the horizontal part is equal to the height of the risers, there is a distinct mismatch between the two current calculation procedures (see also Fig. 15). But from a practical point of view the differences are not crucial so that for practical applications the reflection coefficient method can be used even with smaller $L_{\mathrm{TL}} / h$ ratios.

\section{Conclusion}

In this paper it was shown that cTLT is not sufficient for a finite classical TL with risers at high frequencies. For efficient analysis the TL can be separated into the two riser parts and the asymptotic region. The latter can be handled with cTLT while the riser parts have to be calculated using an advanced TLT, like TLST. The product of matrizants for the three parts finally gives the matrizant for the original whole TL.

Novel reflection coefficients were defined according to the concept of the constant classical ones which are now local and frequency dependent. The current on the TL was calculated using these novel reflection coefficients. For TLs where the horizontal part is significantly larger than the height of the risers the so calculated current fits very well to the ex- act solution using only TLST or a full wave method. Numerical results were shown for several configurations of load impedance or heights of the risers. The result for the current determined via the novel reflection coefficients leads even for small $L_{\mathrm{TL}} / h$ ratios to practically usable values.

The presented method for the analysis of nonuniform TLs is essential for a network theory where such TLs are to be handled. It could be shown that junctions are allowed in regions which show nearly classical behavior for an otherwise nonuniform TL. The formulas for the novel reflection coefficients possess exactly those type of poles, which are necessary for the singularity expansion method (SEM) analysis of the basic frequencies of a TL system. The extension of SEM for nonuniform TL will be of interest for future work.

Edited by: F. Gronwald

Reviewed by: two anonymous referees

\section{References}

Brüns, H., Freiberg, A., and Singer, H.: CONCEPT-II Manual of the Program System, user manual, Technische Universität HamburgHarburg, 2011.

Gantmacher, F.: The theory of matrices, Chelsea Publishing Company, New York, 1984.

Haase, H.: Full-Wave Interactions of Nonuniform Transmission Lines, in: Res Electricae Magdeburgenses (MAFO Vol.9), edited by: Nitsch, J. and Styczynski, Z., Magdeburg, 2005.

Haase, H. and Nitsch, J.: Full-wave transmission-line theory (FWTLT) for the analysis of three dimensional wire-like structures, in: Proc. 14th International Zurich Symposium and Technical Exhibition on Electromagnetic Compatibility, 235-240, Zurich, Switzerland, 2001.

Haase, H., Nitsch, J., and Steinmetz, T.: Transmission-Line Super Theory: A New Approach to an Effective Calculation of Electromagnetic Interactions, The Radio Science Bulletin, 307, 33-60, 2003.

Nitsch, J. and Tkachenko, S.: High-Frequency Multiconductor Transmission-Line Theory, Found. Phys., 40, 1231-1252, 2010.

Nitsch, J., Gronwald, F., and Wollenberg, G.: Radiating Nonuniform Transmission-Line Systems and the Partial Element Equivalent Circuit Method, Wiley, Chichester, West Sussex, UK, 2009.

Rambousky, R., Nitsch, J., and Garbe, H.: Analyzing Simplified Open TEM-Waveguides using Transmission-Line Super Theory, in: International Symposium on Electromagnetic Compatibility, EMC EUROPE 2012, 1-6, Rome, Italy, 2012.

Rambousky, R., Nitsch, J., and Garbe, H.: Application of the Transmission-Line Super Theory to Multiwire TEM-Waveguide Structures, IEEE Trans. EMC, 55, 1311-1319, $2013 \mathrm{a}$.

Rambousky, R., Nitsch, J., and Garbe, H.: Matching the termination of radiating non-uniform transmission-lines, Adv. Radio Sci., 11, 259-264, doi:10.5194/ars-11-259-2013, 2013 b. 